MIT-CTP-4425

UCB-PTH-12/19

\title{
A Note on (No) Firewalls: The Entropy Argument
}

\author{
Yasunori Nomura and Jaime Varela \\ Center for Theoretical Physics, Laboratory for Nuclear Science, and Department of Physics, \\ Massachusetts Institute of Technology, Cambridge, MA 02139, USA \\ Berkeley Center for Theoretical Physics, Department of Physics, \\ and Theoretical Physics Group, Lawrence Berkeley National Laboratory, \\ University of California, Berkeley, CA 94720, USA
}

\begin{abstract}
An argument for firewalls based on entropy relations is refuted.
\end{abstract}


Recently, Almheiri, Marolf, Polchinski, and Sully (AMPS) have posed an interesting paradox [1]: the equivalence principle for an infalling observer is incompatible with the hypothesis that the formation and evaporation of a black hole, as viewed from a distant observer, is described by unitary quantum mechanics and that physics outside the stretched horizon is well approximated by a semi-classical theory (the complementarity hypothesis [2]). If true, this would have profound implications for physics of spacetime and gravity. In particular, AMPS advocate that the most conservative resolution of the paradox is that for an old (but still macroscopic) black hole, the infalling observer hits a "firewall" of high energy quanta at the horizon - a drastic deviation from the prediction of general relativity.

AMPS present two arguments for firewalls: one based on a measurement of early Hawking radiation by an observer falling into an old black hole and the other based on properties of entropies associated with various subsystems of an old black hole. In previous papers, we, together with Sean Weinberg, have refuted the first AMPS argument [3, 4]. (For other work on firewalls, see, e.g., [5].) A key observation is that a full quantum state, to which the unitarity argument applies, is in general a superposition of different classical worlds; on the other hand, general relativity (or the equivalence principle) applies to each of these classical worlds, not necessarily to the whole quantum state. In this short note, we show that a similar observation also allows us to avoid the second AMPS argument, i.e. the argument based on entropies.

Let us briefly recall how the first AMPS argument was addressed in Refs. [3, 4]. The argument is concerned about a state of an old black hole (after the Page time) that has formed from collapse of some pure state:

$$
|\Psi\rangle=\sum_{i} c_{i}\left|\psi_{i}\right\rangle \otimes|i\rangle
$$

where $\left|\psi_{i}\right\rangle \in \mathcal{H}_{\text {rad }}$ and $|i\rangle \in \mathcal{H}_{\text {horizon }}$ represent degrees of freedom associated with early Hawking radiation and the horizon region, respectively. Now, since $\operatorname{dim} \mathcal{H}_{\text {rad }} \gg \operatorname{dim} \mathcal{H}_{\text {horizon }}$, one can construct an operator $P_{i}$ that acts only on $\mathcal{H}_{\text {rad }}$ (not on $\mathcal{H}_{\text {horizon }}$ ) but selects a term in Eq. (1) corresponding to any state $|i\rangle \in \mathcal{H}_{\text {horizon }}: P_{i}|\Psi\rangle \propto\left|\psi_{i}\right\rangle \otimes|i\rangle$. Suppose we choose $P_{i}$ so that $|i\rangle$ is an eigenstate of the number operator, $b^{\dagger} b$, of a Hawking radiation mode that will escape from the horizon region. The state $|i\rangle$ then cannot be a vacuum for the infalling modes $a_{\omega}$, which are related to $b$ by $b=\int_{0}^{\infty} d \omega\left(B(\omega) a_{\omega}+C(\omega) a_{\omega}^{\dagger}\right)$ with some functions $B(\omega)$ and $C(\omega)$. AMPS argue that the fact that one can choose such $P_{i}$ implies that the infalling observer must encounter high energy modes, i.e. the firewall, because he/she can (in principle) measure early Hawking radiation to select the particular term $\left|\psi_{i}\right\rangle \otimes|i\rangle$ in $|\Psi\rangle$.

This argument, however, misses the fact that the existence of projection operator $P_{i}$ does not mean that a measurement performed by a classical observer, which general relativity is supposed to describe, picks up the corresponding state $|i\rangle$. In fact, quite generally, the state $\left|\psi_{i}\right\rangle$ that is entangled with a $b^{\dagger} b$ eigenstate $|i\rangle$ is a superposition of states $\left|\hat{\psi}_{a}\right\rangle$ having well-defined classical 
configurations of Hawking radiation quanta:

$$
\left|\psi_{i}\right\rangle=\sum_{a} d_{i a}\left|\hat{\psi}_{a}\right\rangle
$$

with $d_{i a} \not \delta_{i a}[3]$. An important point is that the coefficients $d_{i a}$ are determined dynamically by the form of Hamiltonian, especially its local nature - it is not something we can choose arbitrarily, e.g. as $d_{i a}=\delta_{i a}$, independent of the dynamics. Substituting Eq. (2) into Eq. (1), we obtain

$$
|\Psi\rangle=\sum_{a}\left|\hat{\psi}_{a}\right\rangle \otimes\left(\sum_{i} d_{i a} c_{i}|i\rangle\right) \equiv \sum_{a}\left|\hat{\psi}_{a}\right\rangle \otimes|\hat{a}\rangle
$$

implying that $|\Psi\rangle$ is a superposition of terms having well-defined configurations of Hawking quanta. Now, a classical world can be defined as a basis state in which the information is amplified; see, e.g., [6, 7]. In Ref. [3], it was considered that the natural basis in the present context, i.e. in addressing AMPS's first argument, is given by interactions between Hawking quanta and the classical measuring devise, which is spanned by the $\left|\hat{\psi}_{a}\right\rangle$ 's. General relativity says that the horizon state in each classical world must be approximately a vacuum state for the infalling modes, and this is not a contradiction since $|\hat{a}\rangle$ can be far from an eigenstate of $b^{\dagger} b$ : $|\hat{a}\rangle \not|i\rangle .1$

We now turn to the main theme of the present note. We suggest that the resolution of the firewall paradox may lie in the emergence of classical worlds in a full quantum state, and this can be determined by the internal dynamics of the horizon (when the system is viewed from outside). (The role played by interactions of a device with early Hawking quanta can be minor.) In particular, the fallen object is represented differently at the microscopic level in each of these classical worlds, although they all correspond to the object falling in the same infalling vacuum when described in general relativity. We will see that this can address AMPS's second argument based on entanglements (and their first argument as well).

AMPS's second argument goes as follows. Consider three subsystems of an old black hole $A$, $B$, and $C$. In an infalling frame, take

$A$ : early/distant Hawking modes,

$B$ : outgoing modes localized near outside of a (small) patch of the horizon,

$C$ : modes inside the horizon that are Hawking partners of $B$.

In a distant frame, the interpretation of $C$ (but not of $A$ or $B$ ) changes:

$C$ : a subsystem of the degrees of freedom composing the stretched horizon,

\footnotetext{
${ }^{1}$ If we prepare a carefully-crafted quantum device that will be entangled with one of the $|i\rangle$ 's and send, e.g., a particle toward the horizon, then that particle may see a firewall. Such a device, however, needs to be an exponentially fine-tuned superposition state of different classical configurations, and can be ignored under realistic circumstances [4].
} 
although it still represents the same degrees of freedom as in Eq. (6) (complementarity). Now, unitarity says that for an old black hole the entropy of the distant modes decreases, implying

$$
S_{A B}<S_{A}
$$

where $S_{X}$ represents the von Neumann entropy of system $X$. On the other hand, the equivalence principle applied to a freely falling observer says

$$
S_{B C}=0,
$$

implying $S_{A B C}=S_{A}$. These two relations contradict strong subadditivity of entropy

$$
S_{A B}+S_{B C} \geq S_{B}+S_{A B C}
$$

since they lead to $S_{B}<0$ if both are true. This implies that one must abandon either unitarity of a black hole formation/evaporation process (with physics outside the stretched horizon well described by a semi-classical theory) or the equivalence principle.

What can be wrong with this argument? Again, the key observation is that unitarity is a statement about an entire quantum state while the equivalence principle is a statement about a classical world - a component/branch of the entire quantum state. Let us assume that both these statements are correct. Then, for the entire quantum state, Eq. (8) must apply while Eq. (9) need not (and in fact cannot) be satisfied. On the other hand, if we focus only on a single component of the state corresponding to a classical world, then the relation as in Eq. (9) must be satisfied (where the entropy should be derived only from that particular component of the state, which we will denote as $\tilde{S}_{B C}$ from now on), while the bound as in Eq. (8) ), i.e. $\tilde{S}_{A B}<\tilde{S}_{A}$, will not be true. This is consistent because the information is (almost) always lost for a classical observer in a quantum mechanical system.

To illustrate this point more explicitly, let us consider the quantum state of an old black hole, which we write in the form

$$
|\Psi\rangle=\sum_{i, j, k, l} c_{i j k l}\left|A_{i}\right\rangle\left|B_{j}\right\rangle\left|C_{k}\right\rangle\left|D_{l}\right\rangle,
$$

where $\left|B_{j}\right\rangle$ and $\left|C_{k}\right\rangle$ represent states for $B$ and $C$ in Eqs. (5, 6, 7). (Here and below, we omit the direct-product symbol.) $\left|D_{l}\right\rangle$ are states for subsystem $D$ which represents all the internal (or stretched horizon) degrees of freedom other than $C$, and $\left|A_{i}\right\rangle$ are states for $A$, in which we now include all the outside degrees of freedom other than $B$. The state $|\Psi\rangle$ comprises our entire quantum state 2

\footnotetext{
${ }^{2}$ To be precise, $|\Psi\rangle$ may not be the complete state for an old black hole, which in general is a superposition of various $|\Psi\rangle$ 's corresponding to black holes in different locations and with different spins [4. Therefore, the complete information about the initial state may not be reproduced from a single $|\Psi\rangle$ alone. This aspect, however, is irrelevant for our argument below, since the entropy for distant radiation decreases in $|\Psi\rangle$ (not only in the complete state), i.e. $S_{A B}<S_{A}$ in $|\Psi\rangle$.
} 
Unitarity of the evolution of $|\Psi\rangle$ implies that Eq. (8) must apply. The strong subadditivity relation in Eq. (10) then says that Eq. (9) cannot be true. In fact, to satisfy the relation, $S_{B C}$ must be of order $S_{B}$, so the $B C$ system is far from maximally entangled. Namely, we have

$$
S_{A B}<S_{A}, \quad S_{B C} \not \approx 0 .
$$

Does this mean that general relativity is incorrect, i.e. a freely falling classical observer finds a drastic violation of the equivalence principle at the horizon?

The answer is no. As discussed before, emergence of classical worlds in a quantum mechanical system is controlled by the dynamics, and a quantum state is in general a superposition of these classical worlds. In particular, the dynamics selects a set of natural basis states in which the information is amplified, and with which any classical objects would be entangled. (For a sufficiently large system with a local Hamiltonian, the basis states are those having well-defined configurations in classical phase space, with spreads dictated by the uncertainty principle.) Let us now consider a state representing one of these classical worlds

$$
|\tilde{\Psi}\rangle=z \sum_{i, j, k, l}^{\prime} c_{i j k l}\left|A_{i}\right\rangle\left|B_{j}\right\rangle\left|C_{k}\right\rangle\left|D_{l}\right\rangle,
$$

where the sum runs only over a subset of the $A$ through $D$ states so that $|\tilde{\Psi}\rangle$ corresponds to a (decohered) classical world in $|\Psi\rangle$. (The sum is denoted with prime to emphasize this point, and $z$ is the normalization constant.) We can define von Neumann entropies for subsystems $A, B$, etc. of the state $|\tilde{\Psi}\rangle$ (not of $|\Psi\rangle$ ). We call such entropies branch world entropies and denote them with the tilde: $\tilde{S}_{X}$ for a subsystem $X$ of $|\tilde{\Psi}\rangle$.

The validity of general relativity requires the $B C$ system in $|\tilde{\Psi}\rangle$ to be maximally entangled, and applying the strong subadditivity relation to $|\tilde{\Psi}\rangle$ then leads to the conclusion that the entropy of the combined $A B$ system cannot be lower than that of $A$ :

$$
\tilde{S}_{B C}=0, \quad \tilde{S}_{A B} \nless \tilde{S}_{A} .
$$

In fact, with $\tilde{S}_{B C}=0$, the strong subadditivity relation yields a stronger condition $\tilde{S}_{A B} \geq \tilde{S}_{A}+\tilde{S}_{B}$. Together with another basic inequality of entropy $\tilde{S}_{A B} \leq \tilde{S}_{A}+\tilde{S}_{B}$, this leads to

$$
\tilde{S}_{A B}=\tilde{S}_{A}+\tilde{S}_{B},
$$

i.e. two subsystems $A$ and $B$ are (almost) separable in a classical world in which $\tilde{S}_{B C} \approx 0$ is valid. This implies that a classical observer in $|\tilde{\Psi}\rangle$ cannot see any entanglement between $A$ and $B$.

We now come to our main point: relations in Eq. (12) are not incompatible with those in Eq. (14) - they are relations on two different quantities: entropies for the entire state and branch world entropies. The two are compatible because for an old black hole the coefficients $c_{i j k l}$ in 
Eq. (11) can have significant support spanning different classical worlds (otherwise $|\Psi\rangle \approx|\tilde{\Psi}\rangle$ ) and because the infalling "vacuum" state in the $B C$ region need not be unique (otherwise, the $B C$ state could be factored from $|\Psi\rangle$, making $S_{A B}<S_{A}$ impossible to satisfy). Here, different classical worlds mean different microstates for the $B C$ states that are described as the same semi-classical spacetime in general relativity.

In the true Minkowski space, the vacuum state is believed to be unique. The near horizon region, however, is only Minkowski vacuum-like (i.e. the equivalence principle requires only a small region compared with the black hole to be Minkowski vacuum-like), and there can be many such states because of microscopic degrees of freedom of the black hole. In particular, the vacuum state for each classical branch can differ in which subsystem of the black hole (i.e. $C+D$ ) is identified as $C$ (i.e. the partner modes of $B$ ) and/or how the modes in $B$ are entangled with those in $C$. (General relativity, however, describes all these states as the same infalling vacuum.) Branches having different entanglement structures between $B$ and the black hole degrees of freedom correspond to different decohered classical worlds.

Because the dimension of Hilbert space for the $C D$ system (black hole) is $e^{\mathcal{A} / 4 l_{P}^{2}}$, where $\mathcal{A}$ and $l_{P}$ are the area of the black hole and the Planck length respectively, the number of different classical worlds can be as large as $e^{\mathcal{A} / 4 l_{P}^{2}}$, enough to fully recover unitarity. The basis for the classical states is selected by the internal dynamics of the horizon, which we assume to be maximally-entangled black hole and exterior near-horizon mode states corresponding to the infalling vacuum. Overlaps among these states are extremely small, so they are regarded as different decohered classical worlds. An infalling classical (macroscopic) object will be entangled with states in this basis. In particular, the object entering the horizon is represented by different states of the black hole in various branches, specifically as a small fluctuation around each of the $e^{\mathcal{A} / 4 l_{P}^{2}}$ different vacuum states.

For $\operatorname{dim} \mathcal{H}_{A} \gtrsim \operatorname{dim} \mathcal{H}_{B C}$, one can easily see that $|\Psi\rangle$ can satisfy Eq. (12) while keeping Eq. (14) for each classical state $|\tilde{\Psi}\rangle$. Of course, this does not prove what we have postulated above: (i) unitarity, (ii) the equivalence principle, and (iii) the semi-classical nature of physics outside the stretched horizon - these are still assumptions. It, however, does show that the argument by AMPS is flawed, and that (i), (ii), and (iii) can all simultaneously be true.

Once again, an important point is to realize that states corresponding to well-defined classical worlds are very special in quantum mechanics (most of the states in general Hilbert space are superpositions of different classical worlds) and that general relativity is a theory describing a classical world (whose emergence is controlled by the dynamics of a system), as has been emphasized by the authors both in black hole physics [3, 4] and cosmology (especially the eternally inflating multiverse) [8, 7]. By carefully considering this point, we conclude that complementarity is a consistent hypothesis. A realization of it in which the intrinsically quantum mechanical nature is manifest has been discussed in Ref. [7], where complementarity is interpreted as (a part of) uni- 
tary reference-frame change transformations acting on the covariant Hilbert space. For detailed discussions on this proposal in the context of black holes, see [4].

\section{Acknowledgments}

We thank Jared Kaplan for useful discussions. This work was supported in part by the Director, Office of Science, Office of High Energy and Nuclear Physics, of the US Department of Energy under Contracts DE-FG02-05ER41360 and DE-AC02-05CH11231, by the National Science Foundation under grants PHY-0855653 and DGE-1106400, and by the Simons Foundation grant 230224.

\section{References}

[1] A. Almheiri, D. Marolf, J. Polchinski and J. Sully, arXiv:1207.3123 [hep-th].

[2] L. Susskind, L. Thorlacius and J. Uglum, Phys. Rev. D 48, 3743 (1993) arXiv:hep-th/9306069]; C. R. Stephens, G. 't Hooft and B. F. Whiting, Class. Quant. Grav. 11, 621 (1994) arXiv:gr-qc/9310006.

[3] Y. Nomura, J. Varela and S. J. Weinberg, arXiv:1207.6626 [hep-th].

[4] Y. Nomura, J. Varela and S. J. Weinberg, arXiv:1210.6348 [hep-th].

[5] R. Bousso, arXiv:1207.5192 [hep-th]; L. Susskind, arXiv:1208.3445 [hep-th]; arXiv:1210.2098 [hep-th]; S. D. Mathur and D. Turton, arXiv:1208.2005 [hep-th]; B. D. Chowdhury and A. Puhm, arXiv:1208.2026 [hep-th]; I. Bena, A. Puhm and B. Vercnocke, arXiv:1208.3468 [hep-th]; A. Giveon and N. Itzhaki, arXiv:1208.3930 [hep-th]; T. Banks and W. Fischler, arXiv:1208.4757 [hep-th]; A. Ori, arXiv:1208.6480 [gr-qc]; S. Hossenfelder, arXiv:1210.5317 [gr-qc]; D.-I. Hwang, B.-H. Lee and D.-H. Yeom, arXiv:1210.6733 [gr-qc]; S. G. Avery, B. D. Chowdhury and A. Puhm, arXiv:1210.6996 [hep-th]; K. Larjo, D. A. Lowe and L. Thorlacius, arXiv:1211.4620 [hep-th]; S. Kalyana Rama, arXiv:1211.5645 [hep-th]; D. N. Page, arXiv:1211.6734 [hep-th].

[6] H. Ollivier, D. Poulin and W. H. Zurek, Phys. Rev. Lett. 93, 220401 (2004) arXiv:quant-ph/0307229]; R. Blume-Kohout and W. H. Zurek, Phys. Rev. A 73, 062310 (2006) arXiv:quant-ph/0505031.

[7] Y. Nomura, arXiv:1110.4630 [hep-th].

[8] Y. Nomura, JHEP 11, 063 (2011) [arXiv:1104.2324 [hep-th]]; Astron. Rev. 7, 36 (2012) arXiv:1205.2675 [hep-th]]; Phys. Rev. D 86, 083505 (2012) [arXiv:1205.5550 [hep-th]]. 